\title{
Modelo de ecuaciones estructurales para determinar la intención de emprendimiento de estudiantes de posgrado
}

\author{
IDROVO POVEDA, FERNANDA KATHERINE \\ Universidad Técnica de Ambato (Ecuador) \\ Correo electrónico: ferkath@hotmail.com \\ VERDESOTO VELÁstegui, OSWALdo SANTIAGO \\ Universidad Técnica de Ambato (Ecuador) \\ Correo electrónico: oswaldosverdesoto@uta.edu.ec \\ VALENCIA NÚÑEZ, EDISON ROBERTO
Universidad Técnica de Ambato (Ecuador)
Correo electrónico: edisonrvalencia@uta.edu.ec \\ CóRDOVA, Víctor Hugo \\ Universidad Técnica de Ambato (Ecuador) \\ Correo electrónico: victorhcordova@uta.edu.ec
}

\begin{abstract}
RESUMEN
En el presente trabajo de investigación se aborda, a través de la utilización de estadísticos y la revisión de literatura científica, el estudio de los determinantes de la intención de emprendimiento, tomando como caso de estudio a los estudiantes de posgrado de la Facultad de Ciencias Administrativas de la Universidad Técnica de Ambato, basándose en la Teoría del Comportamiento Planificado propuesto por Ajzen en el año 1991. De esta manera, se identifica cómo en el ámbito formativo, ciertos factores influyen de diferente manera, para generar la intención emprendedora. El estudio realizado permite conocer además las distintas percepciones que tienen los estudiantes acerca del emprendimiento y su tendencia a generar o no, un negocio propio.
\end{abstract}

Palabras clave: actitud emprendedora, conducta emprendedora, control del comportamiento, determinantes de la intención, emprendimiento, espíritu emprendedor, estudiantes de posgrado, intención de emprendimiento, teoría del comportamiento planificado.

Clasificación JEL: C12; I23; M13; M16.

MSC2010: 03F65; 34F05.

Artículo recibido el 23 de octubre de 2018 y aceptado el 19 de septiembre de 2019. 


\title{
Structural equation model to determine the entrepreneurship intention of graduate students
}

\begin{abstract}
In this research work, through the use of statistics and the review of scientific literature, the study of the determinants of entrepreneurship intention is approached, taking as a case study the postgraduate students of the Faculty of Administrative Sciences from the Technical University of Ambato, based on the Theory of Planned Behavior proposed by Ajzen in 1991. In this way, it is identified how in the training field, certain factors influence in different ways, to generate entrepreneurial intention. The study also allows to know the different perceptions that students have about entrepreneurship and their tendency to generate or not, a business of their own.
\end{abstract}

Keywords: entrepreneurial attitude, entrepreneurial behavior, behavior control, determinants of intention, entrepreneurship, entrepreneurial spirit, graduate students, entrepreneurial intention, theory of planned behavior.

JEL classification: C12; I23; M13; M16.

MSC2010: 03F65; 34F05.

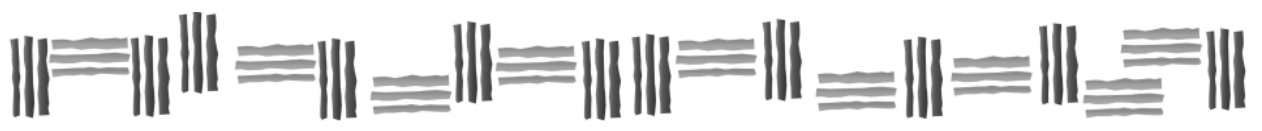




\section{Introducción.}

El emprendimiento es considerado como un aspecto fundamental para el desarrollo económico de un país. Por ello, en los últimos años, se ha tomado un especial interés en su estudio y los factores que lo generan. La comprensión de la conducta emprendedora está relacionada con la intención de emprendimiento y, además, muchos estudios han revelado que la intención, precede al comportamiento emprendedor.

La generación de la conducta emprendedora, se considera esencial para el desarrollo de una nación, ya que, a través de la creación de empresas y fuentes de empleo, se facilita el crecimiento económico. Para que se suscite esta conducta, es necesario que el individuo cuente con la intención y, además, esté centrado en el cumplimiento de un objetivo, lo que influye en las acciones que realice para concretar ese fin.

Para la presente investigación, se tomó como base a la Teoría del Comportamiento Planificado, creada por Ajzen en el año de 1991, que predice adecuadamente las intenciones emprendedoras, analizando la influencia de tres factores: la actitud hacia la conducta, la norma subjetiva y el control sobre la conducta. En el estudio realizado y a través del análisis de estos factores, se pretende conocer cuáles son los determinantes, que influyen en la intención de emprendimiento de estudiantes de posgrado.

El estudio de las variables de actitud, normas subjetivas y control del comportamiento, permite conocer las apreciaciones de los estudiantes sobre la factibilidad de emprender. El presente trabajo tiene como objetivo determinar cuál de las variables independientes, tiene mayor influencia sobre la intención de emprendimiento. A su vez, se busca conocer los dos principales indicadores de cada una de ellas, que contribuirán posteriormente a generar la conducta emprendedora. Para este fin, se utilizará un modelo de ecuaciones estructurales por el método de mínimos cuadrados parciales.

Con los resultados que se obtengan, se diseñará un modelo de determinantes de la intención de emprendimiento que muestre los principales factores que influyen sobre la misma, con la finalidad de potenciarlos en una población determinada.

\section{Antecedentes.}

Para muchas personas la palabra emprendimiento es considerada como un nuevo concepto; sin embargo, ha estado presente a lo largo de la historia de la humanidad. El emprendimiento es la capacidad que tienen los seres humanos de crecer y autosustentarse de una manera novedosa y con ideas renovadas. Ser emprendedor requiere de habilidades no solamente a nivel individual, sino también a nivel colectivo (Jaramillo, 2008).

David McClelland (citado por Marulanda Montoya \& Vélez, p.211), en el año de 1961 expresó que la necesidad de logro es lo que motiva realmente a una persona a convertirse en emprendedor y a su vez, este factor, es en parte el responsable del crecimiento económico. Este autor demuestra en sus investigaciones que este rasgo no es obligatoriamente innato, sino que se puede desarrollar en la persona (Marulanda, Montoya, \& Vélez, 2014).

La diferencia entre un emprendedor y un individuo común la establece su actitud. El emprendedor es la persona capaz de crear, concretar sus ideas, innovar, asumir riesgos y enfrentar problemas. Posee iniciativa propia y sabe crear la estructura necesaria para emprender su proyecto, se comunica y genera redes de comunicación, tiene capacidad convocatoria y de liderazgo (Formichella \& Massigoge, 2004).

Las intenciones de comportamiento constituyen un paso previo a la conducta real, es por ello que se busca explicar la intención de emprender (González et al., 2016). Este análisis se vuelve fundamental 
ya que, según la intencionalidad, se ejecutará el comportamiento emprendedor o creación de una empresa.

Albert Shapero considera que la necesidad de emprender es el resultado de la acción humana y es propia de cada contexto. Identifica una variable independiente, referida al empresario, y una dependiente denominada evento empresarial, que hace referencia a las siguientes características: administración, autonomía, recursos, toma de iniciativa y riesgos. En su modelo, plantea que existen dos grandes percepciones: de conveniencia, que se refiere a las experiencias, valores y son el resultado de la posición de la persona en la matriz de cultura, educación y posición socioeconómica, y la segunda, de factibilidad, considerada como una amalgama de conocimientos, recursos y garantías obtenidos en un negocio o educación formal. Ambas dan origen al proceso de emprendimiento (Parra \& Fleitas, 2010).

Por otro lado, la Teoría de la Expectativa/Valoración atribuida a Víctor Vroom (citado por Marulanda, Montoya \& Vélez, p.212) en 1964, se desarrolló con la finalidad de predecir los niveles de motivación de los individuos. Esta teoría explica la motivación basada en tres tipos de relaciones: (1) probabilidad de que el esfuerzo conducirá a un resultado, (2) lo atractivo de los resultados previstos y (3) la creencia de que un nivel de rendimiento se traducirá en el resultado deseado (Marulanda, Montoya, \& Vélez, 2014)

Por otra parte, en cuanto a las motivaciones para emprender, se destaca el deseo de independencia. El factor económico también es un aspecto determinante (Marulanda \& Morales, 2016). Las tendencias que estimulan al emprendimiento por necesidad son fundamentalmente tres: la necesidad de buscar empleo, la temporalidad del empleo conseguido y la búsqueda de independencia (Morales \& Ariza, 2012). La iniciativa empresarial involucra un cambio cultural que impacta de dos formas: mejorando competencias como liderazgo y comunicación y, a través de un incremento en la innovación y motivación (Leite, Sánchez-Fernández, Leite \& Bezerra, 2015). Para muchos, el espíritu emprendedor depende de la formación que tiene lugar en el hogar durante los primeros años de vida. Ahí, las personas perciben de mejor manera la motivación para autoemplearse en el futuro (Carlos, Contreras, Silva \& Liquidano, 2015).

La Teoría de la Acción Razonada (TRA) constituye un modelo de gran relevancia dentro de la literatura sobre el comportamiento de los sujetos. Se explica sobre la base de la relación entre la actitud y norma subjetiva, lo cual influye en la intención y a su vez, esta última en la conducta. Esta teoría desarrollada por Ajzen y Fishbein (citado por Rueda, Fernandez-Laviada \& Herrero, p.144), en el año de 1980, considera la intención del comportamiento como el mejor previsor de la conducta. De acuerdo con la TRA, el antecedente directo del comportamiento emprendedor, será su intención de desarrollar dicha conducta en el futuro (Rueda, Fernández-Laviada, \& Herrero, 2013). En el año de 1991, Icek Ajzen desarrolla una nueva teoría a partir de la propuesta, complementándola con un nuevo factor y la denomina Teoría del Comportamiento Planificado.

Basado en el modelo de la Teoría del Comportamiento Planificado, la intención de emprendimiento se puede explicar por las actitudes personales y la percepción del control del comportamiento. Como muchos estudios plantean, para los estudiantes la intención está determinada por la percepción de la conveniencia de desarrollar una carrera empresarial. Según otras investigaciones, dicha percepción no viene dada por las normas subjetivas. Ajzen (citado por Osorio \& Londoño, p. 123), en el año de 1987 explicó que este resultado se puede dar cuando las personas tienen un alto nivel de control interno. La búsqueda de independencia y el desarrollo personal también están asociados a la intención de emprendimiento. También puede determinarse por la confianza que tienen los estudiantes en su capacidad de emprender nuevas acciones (Osorio \& Londoño, 2015).

En otros estudios también se han encontrado asociaciones entre la conveniencia percibida, la viabilidad y la tolerancia al riesgo. Estos factores no son independientes entre sí, sino que se encuentran relacionados, como lo propone la TCP (Valencia, Cadavid, Ríos \& Awad, 2012). Tanto la actitud hacia la creación de empresas como la deseabilidad de este comportamiento dependen de la viabilidad 
percibida, mientras que ésta, dependería de la norma subjetiva asociada a un contexto familiar (López \& Ruiz-Ruano, 2014). La educación emprendedora universitaria puede tener efectos significativos en la intención emprendedora. Sin embargo, se desarrolla en estudiantes que poseen ciertas características (Soria-Barreto, Zuniga-Jara \& Ruiz-Campo, 2016).

Para la presente investigación se utilizará el modelo propuesto en la Teoría del Comportamiento Planificado, la cual está científicamente comprobada y ha sido el punto de partida para el desarrollo de estudios a nivel mundial. Los investigadores Krueger y Carsrud, y Krueger y Brazeal (citado por Marulanda, Montoya \& Vélez, p.220) en los años de 1993 y 1994 respectivamente, coincidieron en que la TCP constituye una base sólida en la investigación del emprendimiento. Dos de sus antecedentes actitudinales reflejan la deseabilidad percibida en la realización de una conducta y el control del comportamiento, denota la factibilidad de ejecución de la misma (Marulanda, Montoya, \& Vélez, 2014).

\section{Población y variables.}

\section{Población}

Las unidades de estudio que se utilizarán corresponden a los estudiantes de posgrado de la Facultad de Ciencias Administrativas de la Universidad Técnica de Ambato.

Para la presente investigación se ha considerado necesario trabajar con la totalidad de las unidades de estudio anteriormente descritas, es decir, con la población de estudiantes de posgrado de la Facultad de Ciencias Administrativas, considerando para el efecto a 122 estudiantes que se encuentran matriculados y asistiendo a clases.

\section{Variables}

El proyecto de investigación va a hacer uso de las variables actitud, norma subjetiva, control del comportamiento e intención de emprendimiento. La actitud que tiene una persona hacia una conducta determinada, refleja el grado en que está valorada de manera positiva o negativa. La actitud se determina por las creencias que la unen con resultados y la evaluación de los mismos. Se adquieren cuando se genera una creencia hacia una persona o un objeto. Las actitudes influyen directamente en la toma de decisiones ya que denotan una acción del individuo ante una oportunidad o estímulo (Guzmán, García, Rodríguez \& Alonso, 2014).

Un segundo elemento expone que el individuo se ve condicionado por las expectativas que existen por parte de las demás personas, en relación a su comportamiento. Las normas sociales generan ciertos sentimientos en el sujeto, que impulsan o reducen el deseo de realizar una conducta (Ventura \& Quero, 2013). La norma subjetiva es un razonamiento que pone de manifiesto la presión social percibida por el sujeto que se dispone a ejecutar una conducta. Está determinada por dos componentes: la percepción de aprobación de personas importantes para el individuo y la motivación de éste para acomodarse a los deseos de esas personas (Guzmán, García, Rodríguez \& Alonso, 2014).

Como tercer factor, el control del comportamiento se define como la percepción que tiene una persona sobre la capacidad de controlar las dificultades que se presentan al momento de realizar una determinada conducta (Guzmán, García, Rodríguez \& Alonso, 2014). Depende de la percepción que tiene un individuo sobre su habilidad para llevarla a cabo. El control conductual está determinado por otros factores como son la autoeficacia, los recursos y las creencias de control. Ellos son determinantes para la consecución de un determinado comportamiento u objetivo (Osorio \& Londoño, 2015).

La intención se puede definir como la inclinación de la voluntad hacia un determinado propósito. La intención de emprendimiento consiste en el auto-reconocimiento de la convicción de crear un negocio y la planificación consciente para su realización en un tiempo futuro. La cultura del individuo, 
el sistema de valores y su entorno social pueden conformar el deseo o no, de crear un negocio propio, es decir, de emprender (Soria-Barreto, Zuniga-Jara \& Ruiz-Campo, 2016). La existencia de la intencionalidad de emprendimiento constituye un elemento principal para que se produzca el comportamiento emprendedor. Esto conlleva a la creación de empresas y al crecimiento económico.

\section{Metodología.}

Los modelos de ecuaciones estructurales (Structural Equation Modeling, SEM) son una rama de los modelos estadísticos multivariantes y permiten estimar las relaciones entre múltiples variables (Ruiz, Pardo \& San Martín, 2010). Ayudan a examinar simultáneamente una serie de relaciones de dependencia y los efectos de unas variables sobre otras (Cupani, 2012). Estos modelos tratan de integrar una serie de ecuaciones lineales y establecer cuáles son dependientes o independientes de otras.

La técnica SEM surge de la necesidad de encontrar flexibilidad en los modelos de regresión (Escobedo, Hernández, Estebané \& Martínez, 2016). Son menos restrictivos, ya que permiten incluir errores de medida tanto en las variables de criterio (dependientes) como en las variables predictoras (independientes). Matemáticamente, estos modelos son más complejos que otras técnicas multivariantes como el análisis factorial exploratorio o la regresión (Ruiz, Pardo \& San Martín, 2010).

De manera general, los SEM permiten inicialmente crear modelos del error de medida. Posteriormente, incorporan constructos inobservables (variables latentes y variables teóricas no observadas). Además, confronta el conocimiento a priori e hipótesis con datos empíricos, por lo que estos modelos son más confirmatorios que exploratorios (Escobedo, Hernández, Estebané \& Martínez, 2016).

Con respecto a su estimación, la técnica SEM se basa en la correlación existente entre las variables medidas de una muestra o población, en un momento dado. Los puntos fuertes de estos modelos son: haber desarrollado criterios que permiten su representación gráfica, la posibilidad de concatenar efectos entre variables, permitir relaciones de reciprocidad entre ellas y la posibilidad de confirmar y confrontar hipótesis (Ruiz, Pardo \& San Martín, 2010). Existen dos tipos de indicadores dentro de este modelo: (1) indicadores reflectivos, que dependen de la variable latente y (2) indicadores formativos, que causan a la variable latente (Leyva \& Olague, 2014).

Los especialistas en los modelos SEM coinciden en que, para aplicar esta técnica, se deben cumplir seis fases: (1) la especificación, en donde el investigador establece la relación hipotética entre las variables, (2) la identificación, en la que se determina si un modelo está identificado por una expresión algebraica que lo demuestre, (3) la estimación de parámetros, en donde se determinan los valores de parámetros desconocidos y su error de medición, (4) evaluación del ajuste, que se refiere a la exactitud en los datos del modelo para determinar si es correcto, (5) reespecificación del modelo, para lo que es necesario buscar métodos para mejorar el ajuste del mismo, cambiando los parámetros del original y (6) interpretación de resultados, en donde se aceptan o rechazan las hipótesis planteadas (Escobedo, Hernández, Estebané \& Martínez, 2016).

El principal aporte que realiza SEM es que ayuda al investigador a evaluar modelos teóricos, siendo una de las herramientas más potentes para el estudio de relaciones causales. Estos modelos contribuyen a seleccionar hipótesis causales relevantes, eliminando aquellas no sustentadas por la evidencia empírica (Cupani, 2012).

Para la presente investigación se utiliza el modelo de ecuaciones estructurales por el método de mínimos cuadrados parciales (Partial Least Squares-PLS), teniendo en cuenta que los indicadores son de tipo formativo. La regresión por mínimos cuadrados parciales se utiliza para reducir las dimensiones del conjunto de variables explicativas. Este modelo busca específicamente predecir variables dependientes, por lo que resulta de utilidad para propósitos de análisis causal predictivo. La regresión 
PLS identifica los componentes de la variable dependiente que son los más adecuados para explicar la variable dependiente (Leyva \& Olague, 2014).

\section{Recolección de datos}

El método de la encuesta ha sido aplicado a preguntas que fueron respondidas por los estudiantes de posgrado de la Facultad de Ciencias Administrativas de la Universidad Técnica de Ambato. Se distribuyeron las encuestas a los estudiantes durante sus clases. Se solicitó a los mismos que completaran los cuestionarios en presencia de la investigadora y los devolvieran sin dejar preguntas sin responder. Los encuestados fueron informados sobre el propósito de la investigación y se les aseguró el completo anonimato.

Es importante mencionar que, para la presente investigación, se utilizó el cuestionario de intención emprendedora diseñado y probado por Liñán y Chen, entre los años 2006 y 2009 (citado por Malebana, p.134). El cuestionario fue diseñado exclusivamente para la teoría del comportamiento planificado tal como se aplica al emprendimiento. Esta encuesta se ha aplicado y validado en diversos países desarrollados y en desarrollo (Malebana, 2014).

La encuesta consiste en preguntas basadas en la escala Likert de cinco puntos ( $1=$ Totalmente de acuerdo y $5=$ Totalmente en desacuerdo), una pregunta nominal y una cuantitativa. Las preguntas de tipo escala Likert se usan para la intención empresarial (cuatro ítems), la actitud para convertirse en emprendedor (cinco ítems), el control conductual percibido (siete ítems) y las normas subjetivas (tres ítems), mientras que la escala nominal se utiliza para el sexo ( $1=$ Femenino y $2=$ Masculino $)$ y finalmente la pregunta de tipo cuantitativo, en donde se solicita información sobre el rango de edad del sujeto. Para el análisis de la relación causal entre variables por el método de ecuaciones estructurales por mínimos cuadrados parciales, se utiliza el programa SmartPLS. Los indicadores aparecen en la Tabla 1.

Tabla 1. Indicadores.

\begin{tabular}{cl}
\hline INTENCION_1 & Estoy listo para hacer cualquier cosa para ser un emprendedor. \\
INTENCION_2 & Mi objetivo profesional es ser un emprendedor. \\
INTENCION_3 & Haré todo lo posible para comenzar y dirigir mi propio negocio. \\
INTENCION_4 & Estoy decidido a crear una empresa en el futuro. \\
ACTITUD_1 & Ser emprendedor implica más ventajas que desventajas para mí. \\
ACTITUD_2 & Una carrera como emprendedor es totalmente atractiva para mí. \\
ACTITUD_3 & Si tuviera la oportunidad y los recursos, me gustaría comenzar un negocio. \\
ACTITUD_4 & Entre varias opciones, prefiero ser un emprendedor. \\
ACTITUD_5 & Ser emprendedor me daría una gran satisfacción. \\
CCP_1 & Comenzar un negocio y mantenerlo funcionando sería fácil para mí. \\
CCP_2 & Puedo controlar el proceso de creación de un nuevo negocio. \\
CCP_3 & Tendría un control completo sobre la situación si empiezo y dirijo un negocio. \\
CCP_4 & Estoy dispuesto a hacer cualquier cosa para ser un emprendedor. \\
CCP_5 & Conozco todos los detalles prácticos necesarios para comenzar un negocio. \\
CCP_6 & Si quisiera, podría comenzar y dirigir un negocio fácilmente. \\
CCP_7 & Si tratara de comenzar un negocio, tendría muchas posibilidades de tener éxito. \\
NS_1 & Mis amigos aprobarían la decisión de comenzar un negocio. \\
NS_2 & Mi familia cercana aprobaría la decisión de comenzar un negocio. \\
NS_3 & Mis colegas aprobarían la decisión de comenzar un negocio. \\
\hline
\end{tabular}

Fuente: Elaboración propia. 


\section{Resultados.}

Tabla 2. Resultados del modelo estructural.

\begin{tabular}{ccc}
\hline & Estadísticos t & P Valores \\
\hline ACTITUD $\rightarrow$ INTENCIÓN & 5.643 & 0.000 \\
CONTROL DEL COMPORTAMIENTO $\rightarrow$ INTENCIÓN & 2.514 & 0.012 \\
NORMAS SUBJETIVAS $\rightarrow$ INTENCIÓN & 0.318 & 0.751 \\
\hline
\end{tabular}

Fuente: SmartPLS.

Se analizó el modelo utilizando la técnica bootstrapping, con la que se determina si las relaciones entre variables son o no significativas; se calcula el estadístico t de Student, que debe ser superior a 1,96 para ser considerado válido, al trabajar con un nivel de confianza de $95 \%$. El estudio revela que únicamente dos de las tres variables independientes propuestas influyen sobre la intención de emprendimiento. Para la variable normas subjetivas, los resultados obtenidos a través del software SmartPLS, demuestran que no posee un alto impacto sobre la intención, es decir, no influye en el estado mental del sujeto en el que se encuentra enfocado en dirigir toda su experiencia y acción hacia la creación de un negocio o empresa.

Al evaluar las variables de actitud y control del comportamiento, se observa que las mismas sí influyen directamente sobre la intención de emprendimiento. Por lo tanto, los juicios evaluadores que tienen las personas antes de desarrollar determinado comportamiento, son determinantes al momento de emprender, ya que reflejan la creencia favorable o desfavorable del individuo para crear una empresa. Además, la percepción sobre el grado de control que tiene una persona sobre la disponibilidad de factores que pueden facilitar o impedir el inicio de un negocio, es importante para generar la intención $\mathrm{y}$, por lo tanto, la conducta emprendedora.

Por otra parte, se pudo identificar que la variable normas subjetivas, no posee influencia sobre la variable dependiente, es decir, las creencias que tienen las personas de que otros individuos aprueben o no la realización de un determinando comportamiento, no es relevante al momento de crear un negocio propio, lo que deja de manifiesto que las personas permanecen pendientes de otros factores, antes que de la opinión de terceros.

En concordancia con el resultado obtenido, en otras investigaciones alineadas a la Teoría del Comportamiento Planificado, se reporta un efecto nulo de las normas subjetivas y se demuestra que las actitudes personales y la percepción del control del comportamiento, explican la intención emprendedora en los estudiantes (Osorio \& Londoño, 2015).

\section{Modelo de determinantes de la intención de emprendimiento}

Para el modelo que se propone a continuación, se utilizaron las dos variables independientes que según se demostró, sí tienen influencia sobre la intención de emprendimiento. Además, se tomaron en consideración los dos indicadores con el valor de estadístico t más alto, de los factores de actitud y control del comportamiento y de la intención o variable dependiente.

Para el desarrollo del modelo se utiliza el programa SmartPLS en donde, mediante el análisis de ecuaciones estructurales, se obtienen nuevamente los valores del estadístico t, para comprobar la influencia de las variables.

Los dos indicadores que obtuvieron el valor más alto en cada variable son: 
Actitud:

- ACTITUD_4: Entre varias opciones, prefiero ser un emprendedor.

- ACTITUD_5: Ser emprendedor me daría una gran satisfacción.

Control del comportamiento:

- CCP_4: Estoy dispuesto a hacer cualquier cosa para ser un emprendedor.

- CCP_6: Si quisiera, podría comenzar y dirigir un negocio fácilmente

Intención:

- INTENCION_3: Haré todo lo posible para comenzar y dirigir mi propio negocio.

- INTENCION_4: Estoy decidido a crear una empresa en el futuro.

Tomando como base el estudio realizado, a continuación, se expone el modelo generado. La variable Actitud, es más cercana a la Intención, dado que tiene mayor peso en el confidente de trayectoria, mientras que la variable Control del comportamiento, tiene un valor menor del coeficiente, pero sigue siendo igualmente importante.

Gráfico 1. Modelo estructural propuesto.

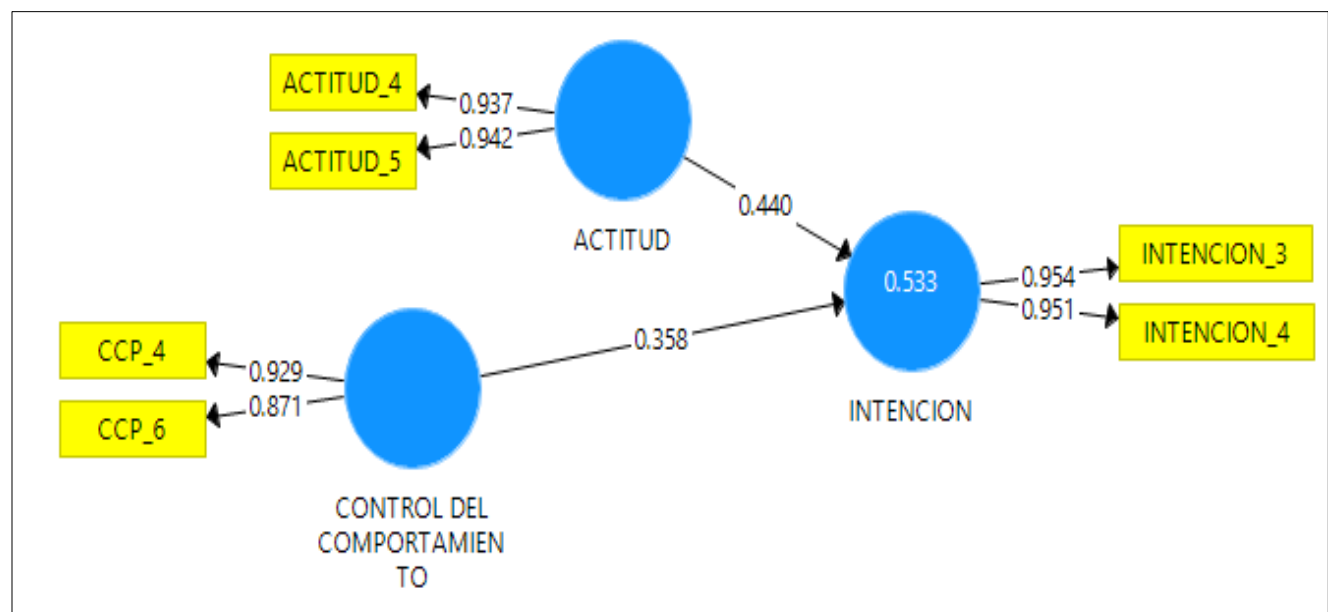

Fuente: Elaboración propia.

Tabla 3. Resultados del modelo estructural propuesto.

\begin{tabular}{ccc}
\hline & Estadísticos t & P Valores \\
\hline ACTITUD $\rightarrow$ INTENCION & 4.331 & 0.000 \\
CONTROL DEL COMPORTAMIENTO $\rightarrow$ & 3.654 & 0.000 \\
INTENCION & & \\
\hline
\end{tabular}

Fuente: SmartPLS. 
Utilizando los mismos criterios expuestos en el primer resultado, en las dos variables independientes, el valor calculado del estadístico t de Student es superior a 1,96, lo que indica que el efecto de la actitud y el control del comportamiento sobre la intención es alto.

\section{Conclusiones.}

Analizando los indicadores que más influyen sobre las variables, se determina lo siguiente:

La afirmación "ser emprendedor me daría una gran satisfacción" (ACTITUD_5), es el indicador que posee mayor peso sobre la variable Actitud, por lo que se concluye que el nivel de satisfacción es importante en la actitud emprendedora.

La afirmación "entre varias opciones, prefiero ser un emprendedor" (ACTITUD_4), es el segundo indicador más influyente sobre la variable Actitud, por lo que se concluye que el grado de inclinación hacia el emprendimiento es importante para generar una actitud de emprendimiento.

La afirmación "estoy dispuesto a hacer cualquier cosa para ser un emprendedor." (CCP_4), es el indicador más relevante sobre la variable Control del comportamiento, por lo que se concluye que la disposición del individuo para empezar una empresa es significativa al momento de evaluar si es o no factible emprender.

La afirmación "si quisiera, podría comenzar y dirigir un negocio fácilmente" (CCP_6), es el segundo indicador más importante sobre la variable Control del comportamiento, por lo que se concluye que el nivel de confianza de una persona es decisivo para que surja la conducta emprendedora.

La afirmación "haré todo lo posible para comenzar y dirigir mi propio negocio" (INTENCION_3) es el indicador más influyente sobre la variable Intención, por lo que se concluye que la perseverancia del individuo es importante para desarrollar la intención emprendedora.

La afirmación "estoy decidido a crear una empresa en el futuro" (INTENCION_4), es el segundo indicador más influyente sobre la variable Intención, por lo que se concluye que el nivel de decisión de una persona influye directamente sobre la intención de generar un negocio propio.

Por otra parte, como se puede apreciar en la tabla, existe una influencia directa de la actitud y el control del comportamiento sobre la intención de emprendimiento.

Para los estudiantes de posgrado de la Facultad de Ciencias Administrativas de la Universidad Técnica de Ambato, la intención está determinada por la percepción de independencia al emprender un negocio propio, la que no está originada en las normas subjetivas. En esta investigación, salen a relucir aspectos actitudinales propios de la etapa en la que se encuentran los estudiantes, como los deseos de desarrollo personal y autonomía laboral. Además, se observan algunos patrones de pensamiento, relacionados con la confianza, perseverancia y predisposición para generar un emprendimiento, mismos que demuestran que las actitudes personales tienen un mayor grado de influencia sobre la intención, antes que el control del comportamiento. 


\section{Referencias}

Carlos, C., Contreras, L., Silva, M., \& Liquidano, M. (2015). El Espíritu Emprendedor y un Factor que Influencia su Desarrollo Temprano. Conciencia Tecnológica, 49, 46-51.

Cupani, M. (2012). Análisis de Ecuaciones Estructurales: conceptos, etapas de desarrollo y un ejemplo de aplicación. Tesis, 1, 186-199.

Escobedo, M., Hernández, J., Estebané, V., \& Martínez, G. (2016). Modelos de Ecuaciones Estructurales: Características, Fases, Construcción, Aplicación y Resultados. Ciencia \& Trabajo, 18(55), 16-22.

Formichella, M., \& Massigoge, J.I. (2004). El concepto de emprendimiento y su relación con la educación, el empleo y el desarrollo local. VII Congreso de Administración.

González, M., Valantine, I., Pérez, C., Aguado, S., Calabuig, F., \& Crespo, J. (2016). La influencia del género y de la formación académica en la intención de emprender de los estudiantes de ciencias de la actividad física y el deporte. Intangible Capital, 12(3), 759-788.

Guzmán, F., García, B., Rodríguez, L., \& Alonso, M. (2014). Actitud, norma subjetiva y control conductual como predictores del consumo de drogas en jóvenes de zona marginal del norte de México. Frontera Norte, 26(51), 53-74.

Jaramillo, L. (2008). Emprendimiento: Concepto básico en competencias. Lumen, 7, 1-6.

Leite, E., Sánchez-Fernández, M., Leite, E., \& Bezerra Correia, E. (2015). El espíritu emprendedor: condicionantes para la innovación. Holos, 5, 278-291.

Leyva, O., \& Olague, J. (2014). Modelo de Ecuaciones Estructurales por el Método de Mínimos Cuadrados Parciales (Partial Least Squares-PLS). En M. Asensi, R. Cotarelo, M. Echenique, J. Fernández, P. Oñate, J. Romero, \& J. Tamayo, Métodos y Técnicas Cualitativas y Cuantitativas Aplicables a la Investigación en Ciencias Sociales. México: Tirant Humanidades.

López, J., \& Ruiz-Ruano, A. (2014). Modelado de la intención emprendedora con redes bayesianas. Revista de Psicología, 23(2), 71-87.

Malebana, J. (2014). Entrepreneurial intentions of South African rural university students: A test of the theory of planned behaviour. Journal of Economics and Behavioral Studies, 6(2), 130-143.

Marulanda, F., \& Morales, S. (2016). Entorno y motivaciones para emprender. Escuela de administración de negocios, 81, 12-28.

Marulanda, F., Montoya, I., \& Vélez, J. (2014). Teorías motivacionales en el estudio del emprendimiento. Pensamiento \& Gestión, 36, 206-238.

Morales, A., \& Ariza, A. (2012). Valores, actitudes y motivaciones en la juventud ante el emprendimiento individual y colectivo. Revista de Estudios Cooperativos, 112, 11-35.

Osorio, F., \& Londoño, J. (2015). Intención emprendedora de estudiantes de educación media: extendiendo la teoría de comportamiento planificado mediante el efecto exposición. Cuadernos de Administración, 28(51), 103-131.

Parra, L., \& Fleitas, M. (2010). Incubación de proyectos para iniciativas empresariales en la Universidad Libre de Cali. Dirección, 31(3), 1-8. 
Rueda, I., Fernández-Laviada, A., \& Herrero, Á. (2013). Aplicación de la teoría de la acción razonada al ámbito emprendedor en un contexto universitario. Investigaciones Regionales, 26, 141-158.

Ruiz, M., Pardo, A., \& San Martín, R. (2010). Modelos de ecuaciones estructurales. Papeles del Psicólogo, 31(1), 34-45.

Soria-Barreto, K., Zuniga-Jara, S., \& Ruiz-Campo, S. (2016). Educación e Intención Emprendedora en Estudiantes Universitarios: Un Caso de Estudio. Formación Universitaria, 9(1), 25-34.

Valencia, A., Cadavid, L., Ríos, D., \& Awad, G. (2012). Factores que inciden en las intenciones emprendedoras de los estudiantes. Revista Venezolana de Gerencia, 17(57), 132-148.

Ventura, R., \& Quero, M. (2013). Factores explicativos de la intención de emprender en la mujer. Aspectos diferenciales en la población universitaria según la variable género. Cuadernos de Gestión, 13(1), 127-149. 